\title{
Rats' memory for serially presented flavors: Effects of interstimulus interval and generalization decrement
}

\author{
PHIL REED \\ University College London, London, England
}

\begin{abstract}
In four experiments, the effect of sequential exposure to a series of five novel flavors on the subsequent neophobic response of water-deprived rats to those flavors when they were presented simultaneously was examined. After a list-test interval of $30 \mathrm{~min}$ and a list-interstimulus interval of $10 \mathrm{sec}$, the rats generally consumed more of the first and last flavors presented in the initial sequence. This finding was taken to reflect the existence of primacy and recency effects. Experiment 1 provided evidence that successive contamination can occur between flavors in the initial list, making subsequent recognition of later flavors in the list more difficult. However, this effect was overcome by presentation of water between each flavor during the list exposure. Experiments 2 and 4 showed that primacy was not a necessary result of successive contamination in this procedure, by demonstrating that increasing the interstimulus interval between list items decreased the size of the primacy effect. This result suggests that rats' memory for serially presented items may be controlled by mechanisms different from those typically implicated in the human verbal memory literature. In Experiment 3, the question of whether the testing procedure adopted here could have introduced sources of artifactually produced serialposition effects was explored, but no such influence was found.
\end{abstract}

Reed, Croft, and Yeomans (1996) investigated rats' memory for serially presented material, using a nonspatial paradigm involving flavors. In this report, the tendency of rats to avoid novel substances (neophobia) was exploited to obtain a measure of their memory for those flavors. To the extent that subjects remember previously having been exposed to a flavor, the subjects should drink more of that flavor when it is again presented to them. The serial presentation of a list of items results, typically, in better memory for the items occupying the initial and terminal portions of the list than for those items occupying the central positions in the list (Baddeley, Papagno, \& Andrade, 1993; Glazner \& Cunitz, 1966). These effects are termed primacy and recency, respectively. Reed et al. (1996) demonstrated that both primacy and recency effects could be obtained in the above-mentioned flavor paradigm. The rats consumed more of the flavors presented at the start and end of the series than of the flavors presented in the middle of the initial sequence.

Although it is not contentious that the serial presentation of items to nonhuman subjects will result in recency effects (Macphail, 1980; Sands \& Wright, 1980; Thompson \& Herman, 1977), the existence of primacy effects in

\footnotetext{
These data were initially discussed at the Experimental Analysis of Behaviour Group Meeting, London. Thanks are due Jennifer Hewitt for help in collecting these data, Todd Schachtman for reading earlier drafts of this report, and Lisa A. Osborne for her support. Correspondence concerning this article should be addressed to P. Reed, Department of Psychology, University College London, Gower Street, London, WC1E 6BT, England (e-mail: p.reed@ucl.ac.uk).
}

all but a few situations has not yet gained universal acceptance (see, e.g., D. Gaffan, 1983; E. A. Gaffan, 1992). It is well established that rats show primacy effects in spatial tasks (e.g., Bolhuis \& Van Kampen, 1988; DiMattia \& Kesner, 1984; Harper, McLean, \& DalrympleAlford, 1993; Reed \& Richards, 1996). However, the evidence for primacy effects with rats in nonspatial tasks is less clear. Reed, Chih-Ta, Aggleton, and Rawlins (1991) presented rats with a series of five items, each located at the end of an arm in a maze. After a retention interval, the rats were given a choice between an arm containing a copy of one of the items from the list and an arm containing a novel item. To earn a reward, the rat had to choose the arm containing the novel item. In this experiment, choice accuracy was better when the discrimination involved an object from the start or end of the list than when it involved an item from the middle of the list. In contrast, Deacon and Rawlins (1995) reported a series of experiments that putatively failed to replicate the result reported by Reed et al. (1991). However, in seven different conditions that tested for serial position effects, Deacon and Rawlins obtained six null results. It should be noted that such null results cannot properly be said to represent a failure to replicate only the study by Reed et al. (1991). These results also fail to replicate other studies with nonhumans that have shown recency effects under such conditions, making interpretation of those findings extremely problematic. In fact, the one interpretable result reported by Deacon and Rawlins was a demonstration of both primacy and recency effects. However, given the controversial nature of the above-mentioned procedure (cf. E. A. Gaffan, 1992; 
Reed, 1994), the demonstration of primacy and recency effects in the flavor paradigm (Reed et al., 1996) is one of the few unequivocal findings of primacy for rats in a nonspatial paradigm. Given this, additional demonstrations of serial position effects with this procedure and an analysis of the possible causes of these effects in this paradigm appear to be warranted.

One set of explanations of serial position effects in humans attributes primacy effects to the rehearsal of early items in the list. This rehearsal allows these items access to a permanent memory store, from which recall is highly likely. On this view, recency is attributed to the retrieval of the later items in the list from a temporary short-term store, in which the last items are still held (Atkinson \& Shiffrin, 1968). Alternatively, another set of theories employs the notion of distinctiveness as an explanation for serial position effects in humans (see Johnson, 1991; Neath, 1993; Schmidt, 1991). Primacy and recency may be the product of such distinctiveness, since the early and late items in the list, owing to their positions, are relatively free from interference by the other items in the list (Johnson, 1991; Wright, Santiago, Sands, Kendrick, \& Cook, 1985).

It is yet to be established which of these putative mechanisms is operative in the nonhuman case. Of equal importance, it may be that nonhuman serial position effects are generated by processes different from those operative in humans. For example, serial position effects in nonhumans may be the result of the relative hedonic properties of the items occupying various positions in the list of stimuli (Wixted, 1986; Wynne, 1995). If different processes support serial position effects in humans and in other species, it may be that the same manipulation will have different effects on memory for serially presented information. In fact, there are examples of differences between humans and nonhumans with respect to the effects of various manipulations on memory for serially presented material: Varying the interstimulus interval (ISI) between items in a list increases the size of the primacy effect in human subjects (Glazner \& Cunitz, 1966), as long as the items are nameable (Reed, 2000; Wright, Cook, Rivera, \& Shyan, 1990). In contrast, this manipulation apparently reduces the size of the primacy effect in monkeys (Cook, Wright, \& Sands, 1991). Cook et al. manipulated the viewing time and the ISI experienced by two monkeys in a list-learning procedure. The increase in ISI led to a decrease in overall accuracy in identifying the list items on the part of the monkeys and gave the impression that the primacy effect was reduced. This is a potentially important point deserving further investigation in a procedure ther than that used by Cook et al., in order to confirm its generality across nonhuman species and test situations.

It is possible that the results obtained by Reed et al. (1996), using the flavor paradigm, reflect processes ther than those typically implicated in the generation of serial position effects, either in humans or in nonhunans. In order to demonstrate that the flavor paradigm is i valid index of memory for serially presented items, ma- nipulations would need to influence memory similarly in this procedure and in other procedures. It could be, for example, that the flavor paradigm generates serial position effects via nonmemorial processes. Potentially, one of these processes involves generalization decrement between the initial presentation of the flavors during list exposure and the re-presentation at test. The sequential presentation of flavors during the list exposure phase may lead to one flavors contaminating the next flavor in the sequence. If successive contamination between flavors occurs, the only item that would be familiar to the rat at test would be that flavor presented first in the initial list; all of the other flavors would effectively have been presented as compound stimuli. If this were so, the first flavor would be the only one free from a neophobic response during the test (since it would be the only one recognized as having been consumed previously), and a primacy-like effect would be seen. This possibility was noted by Reed et al. (1996), but its influence on performance was not systematically assessed. If the present flavor paradigm is to be established as reflecting the operation of mechanisms responsible for serial-memory effects, the above generalization decrement account has to be shown to be an insufficient explanation of the results. If the above ISI manipulations were found to have the same effects on recognition for serially presented material in the present paradigm that they have in other procedures (i.e., primacy could be attenuated by increases in the ISI), it could be demonstrated that the primacy effect is not a necessary consequence of the present procedure.

Thus, the present series of studies was devised with three aims: first, to replicate the original serial position effects noted by Reed et al. (1996); second, to extend the findings of Cook et al. (1991) concerning the effect of the ISI manipulation on serial position functions; and third, in the process of investigating the effect of the ISI on memory for serially presented items, to demonstrate that serial position functions generated in the present flavor paradigm are not a consequence of paradigm-specific processes.

\section{EXPERIMENT 1}

In Experiment 1, the possibility that generalization decrement plays a role in the generation of primacy effects was examined. Successive exposure to flavors may lead to contamination of the later flavors by earlier flavors, reducing the possibility of recognition of the contaminated items at test. Previously, this problem was addressed by the presentation of water between each flavor (Reed et al., 1996). However, no evidence was presented that such water presentations between the flavors during exposure made any difference to the pattern of performance at test.

Even if such a procedure did alter performance, it is unclear whether the presence of water during the ISI between the flavors or the ISI per se was critical in producing the effects observed. In Experiment 1, these possibil- 
ities were addressed by assessing the influence on serial position effects when both water and an ISI, just an ISI, or neither water nor an ISI was presented during list exposure. Should there be a significant degree of generalization decrement in the absence of water during the ISI, primacy-like effects should be most pronounced in the group receiving no ISI and no water; in this group, there is maximum potential for interstimulus interference. If interstimulus interference is reduced by intervening water, the role of the water and the ISI per se will be assessed by comparison of the two further groups. If the presentation of water is critical, only the group with water should show a recency effect. If the presence of an ISI is sufficient in itself to reduce stimulus interference, both of the groups with an ISI, irrespective of the presence of water, should show a recency effect.

\section{Method}

Subjects. Seventy-five male hooded Lister rats were used (plus 8 animals for a pilot study). The rats were 5-6 months old and had a free-feeding body weight range of $485-615 \mathrm{~g}$. The rats had all experienced standard laboratory diet and Noyes reinforcement pellets, but none had experience with the flavors to be used in the present experiment. The subjects were housed singly, with free access to food in their home cages. Prior to testing, the rats were water deprived for between 16 and $20 \mathrm{~h}$. The rats were on a 12:12-h light:dark cycle, and water deprivation was initiated $3 \mathrm{~h}$ before dark onset.

Apparatus. The subjects were all housed individually in plastic cages with metal roofs $(40 \times 25 \times 20 \mathrm{~cm})$, with sawdust on the cage floors. Flavors were presented via small plastic bottles with metal spouts that could be inserted through the bars of the cage. The flavors were all locally obtained, commercially produced food flavorings (U.K. Safeway Brand), which were diluted with tap water. The bottles were filled with $50 \mathrm{~g}$ of tap water, and then 15-20 drops of a food flavoring were added to the water. (This amount was enough to make the solution have a distinct odor, at least to a human nose.) The flavors used were lemon, vanilla, almond, peppermint, and rum. A number of 2-ml syringes (one for each flavor in the experiment) were also used in this study.

Procedure. Prior to the study proper, a small pilot study $(n=8)$ was conducted in order to ensure that the flavors would be consumed in roughly equivalent amounts. Two 4-h water-deprived subjects were given 15-min access to all five of the above flavors simultaneously. After this time, the amount drunk of each of the flavors was measured. If both rats tended to consume large amounts of a particular flavor, an attempt was made to equate future consumption by either diluting or concentrating the amount of flavoring present in the liquid. The new concentration was given to 2 new rats, and the procedure was repeated until there were no systematic preferences for any of the flavors.

For the critical experimental manipulation, the subjects were moved from the colony room into a test room, in which all deprivation and testing was conducted. On 2 consecutive days prior to the presentations of the flavors, all the subjects were water deprived for $4 \mathrm{~h}$. The rats were then presented with the nozzles of 2-ml syringes filled with water and inserted into their home cages. The rats were allowed to drink from those syringes. This procedure entailed allowing the animals' mouths to make contact with the syringes and slowly depressing the syringes to provide constant, slow flows of liquid that the animals could drink. Over the course of these 2 days, the subjects were habituated to the infusion procedure. All the rats readily learned to drink in this manner. For the flavor exposure, the subjects were presented with five different, novel flavors, using the infusion technique. The syringe was inserted into the rats' cages for $30 \mathrm{sec}$, during which $0.5 \mathrm{ml}$ of liquid was delivered to the animals

The subjects were divided into three groups $(n=25)$, and 1 animal from each group was matched with 1 animal from each of the other two groups during the exposure phase. The rats in each matched triplet ( 1 from each group of subjects) received the flavors in the same serial order during the exposure phase of the study. The order of flavor presentation was varied across the 25 rats in each group (1 rat from each of the three groups received the same order of flavor presentations as a rat in each of the other two groups) to control for any remaining flavor preferences. Each flavor occurred at each serial position in the initial list five times. The order of the five flavors was also quasi-random between rats, in that no runs of three flavors were ever given to 2 rats. Prior to the first flavor, the subjects in Group ISI-Water received $0.5 \mathrm{ml}$ of water, delivered over $30 \mathrm{sec}$ by the infusion technique. Between each flavor, the subjects received $0.2 \mathrm{ml}$ of water, delivered over $10 \mathrm{sec}$ by the same infusion technique. The subjects in Group ISI were treated identically to those above but had no water presented between the flavors. The subjects in Group No were presented with the sequence of flavors, but had a minimal ISI (approximately $2-3 \mathrm{sec}$ ) between the flavors and had no water presented between the flavors.

Following exposure, all the rats received a 30 -min retention interval ( $90 \mathrm{sec}$ water plus $28.5 \mathrm{~min}$ without liquid). At test, all five of the bottles were replaced through the front of the subjects' cages. The order in which the bottles were inserted from left to right across the cages was random with respect to the order in which the subjects had experienced the flavors during the exposure phase. The subjects were then allowed 15 min undisturbed free-access to the solutions.

\section{Results and Discussion}

Figure 1 displays the mean amount consumed at each serial position, during the test phase, for all three groups in Experiment 1. An inspection of the amount of liquid consumed during test reveals that larger amounts of the initial flavor, relative to those in the middle of the sequence, were drunk in all three groups. However, only in Group ISI-Water was there a recency effect.

A two-factor analysis of variance (ANOVA), with group as a between-subjects factor and serial position as a withinsubjects factor, was conducted on these data. A rejection criterion of $p<.05$ was adopted for this and all subsequent analyses. This analysis revealed no significant effect of group $(F<1)$, a significant main effect of serial position $[F(4,288)=5.26]$ and a significant interaction between these factors $[F(8,288)=3.26]$. Trend tests were conducted on these data, which revealed the only significant trend in Group ISI-Water to be quadratic $[t(24)=$ 2.03]. That is, the test data for Group ISI-Water conformed to a U-shaped pattern. This pattern would be predicted if the rats had consumed, at test, more of the first and last flavors presented in the initial list than they had consumed of the middle flavors. Planned comparisons revealed that there was, indeed, a significant difference between the amount of flavor consumed, at test, at serial position one and serial position three $[t(24)=2.07]$, and similarly, the difference between serial positions three and five was significant $[t(24)=2.01]$. In contrast, only the linear trends were significant in the other two groups [smallest $t(24)=2.771$. On inspection of the data, this result implied that there was a significant decreasing trend in 


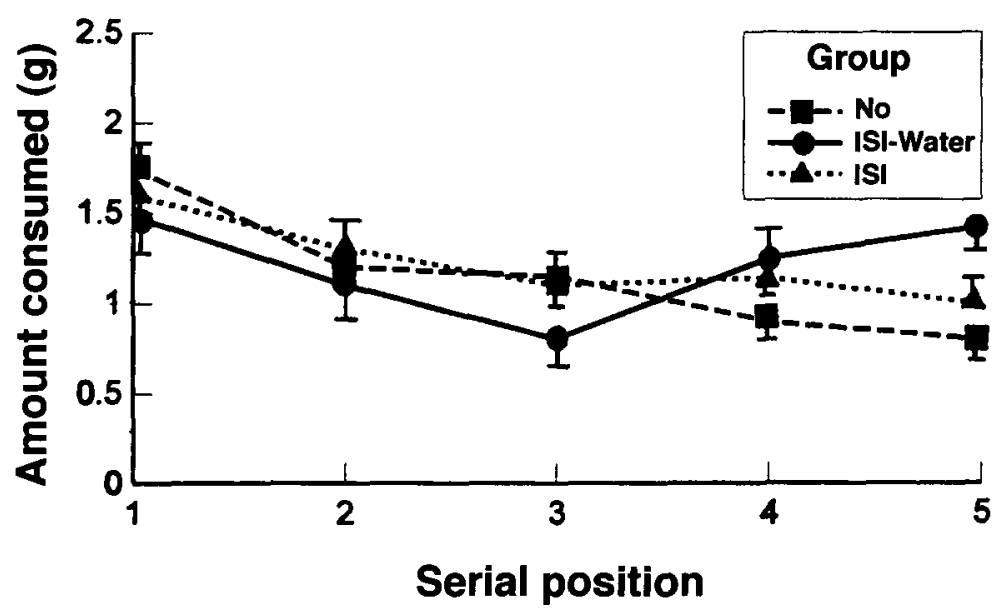

Figure 1. Results from Experiment 1: mean amount of liquid (in grams) consumed during test. Error bars are standard errors of the mean. Group ISI-water, water presented during a 10-sec interstimulus interval (ISI); Group ISI-no water, no water presented during a 10-sec ISI; Group No, no ISI presented during list exposure.

the data across the serial positions for both Group ISI and Group No. Planned comparisons for both of these groups revealed that serial position one differed significantly from serial position three [smallest $t(24)=2.55$ ], but that serial position three did not differ from serial position five (both $p s>.20$ ). Simple effect comparisons between the consumption of the liquids occupying the various positions revealed no differences between the groups, except for serial position five, in which Group ISI-Water consumed more liquid than did either of the other two groups $[F(2,354)=2.98]$.

A consideration of the data from Group ISI-Water, reveals that primacy and recency effects can be obtained with this procedure, and this replicates the results reported by Reed et al. (1996). The generalization decrement account suggests that test consumption of the first flavor presented in a sequence should be large, relative to consumption of the subsequent flavors. It does not suggest that consumption of the final flavor (i.e., a recency effect) should be large. That recency effects were replicated in the present experiment confirms that generalization decrement is unlikely to be the sole mechanism responsible for the present data.

The conclusion above is strengthened by an examination of the different performances of the three groups. When water was presented between the flavors, the subjects drank more of the last flavor in the series than they did when no water was presented between the flavors. This result implies that, within the limits tested in the present experiment, it is the presence of water, and not the ISI per se, that is responsible for the increase in consumption of the final flavor, relative to when no water is presented during the present ISI. The reduction in consumption of the last item, relative to the other items, when a 10-sec ISI but no water was presented during exposure to the novel flavors is consistent with the suggestion that the inter- stimulus water served to prevent interference between flavors during exposure to the series. In the absence of water presented during the $10-\mathrm{sec}$ ISI, there could be interference between the flavors in the list, resulting in generalization decrement at test. In total, these results suggest that perceptual interference may be operating in the present experiment but that, given the appropriate control procedures, this is not a sufficient explanation of the results. Of course, it could always be the case that generalization decrement was operating in all three groups but was only powerful enough to obscure the findings of primacy when a mouthwash was not given to the animals. This possibility cannot be discounted, although it would have to be accepted that some aspect of the procedure, in addition to generalization decrement, was also operating to promote the difference in serial position recognition effects noted in the present study.

\section{EXPERIMENT 2}

Although the results above are consistent with the suggestion that generalization decrement does not play the sole role in generating serial position effects, they do not completely rule out this possibility. In order to do so, it would be necessary to show that recency effects could be obtained in the absence of any primacy effects. An inspection of the data presented in Experiment 1 suggests that the group receiving no ISI displayed a numerically greater (although not statistically significant) primacy effect than did those subjects receiving an ISI. Previous demonstrations of the effect of increasing the ISI on nonhuman memory for serially presented items have shown a similar tendency. Cook et al. (1991) reported data consistent with the suggestion that a longer ISI between list items reduces the size of the primacy effect in monkeys, whereas recency remains intact. This is a potentially im- 


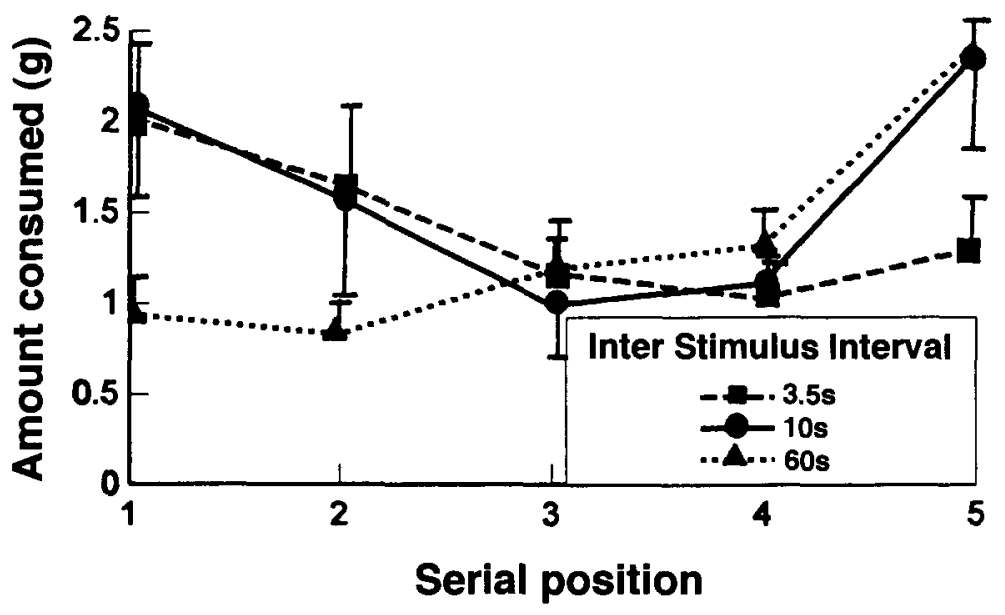

Figure 2. Results from Experiment 2: mean amount of liquid (in grams) consumed during test for all three groups. $3.5 \mathrm{~s}, 3.5 \mathrm{sec}$ interstimulus interval (ISI); 10s, 10-sec ISI; 60s, 60-sec ISI. Error bars are standard errors of the mean.

portant finding, since it is not typically found in human subjects, at least when these subjects are presented with verbal material (see Glazner \& Cunitz, 1966). A replication of this effect of ISI would be of some theoretical significance, and it would also serve to show that primacy effects are not a necessary product of the flavor procedure that is caused by generalization decrement.

The aim of the second experiment was to investigate the effects of increasing the length of the ISI between list items on rats' memory for serially presented flavors. Three groups of rats were studied. One group received a minimal ISI (approximately $3.5 \mathrm{sec}$ ), and a second group received an ISI of $10 \mathrm{sec}$, both as described in Experiment 1. The third group received an ISI of $60 \mathrm{sec}$. If a longer ISI does produce lower levels of primacy with the present procedure, the group receiving the longer ISI should display a reduced primacy effect, relative to the former two groups. Such a result would also help to validate the flavor procedure as one that taps central memory processes, rather than one that generates apparent serial position effects through paradigm-specific processes.

\section{Method}

Subjects and Apparatus. Seventy-five male hooded Lister rats were used. The rats were $8-9$ months old and had a free-feeding body weight range of $495-745 \mathrm{~g}$. The rats had similar previous experience and were maintained as described in Experiment 1. The apparatus and flavors were the same as those in Experiment 1 .

Procedure. A small pilot study was conducted as described in Experiment 1. The subjects were habituated to the infusion procedure in the same way as those in Experiment 1. For the critical experimental manipulation, all the groups of rats were treated like Group ISI-Water in Experiment 1, with the following exceptions. Group $3.5 \mathrm{sec}$ had an ISI of $3.5 \mathrm{sec}$, which involved the presentation of approximately $2 \mathrm{sec}$ water between each flavor. This resulted in there being less time to drink the interstimulus water in this group, relative to the other two groups, which received a longer ISI. Group $10 \mathrm{sec}$ received the same treatment as Group ISI-Water in Experiment 1. Group $60 \mathrm{sec}$ received the same treatment as Group
$10 \mathrm{sec}$, except that after the $10 \mathrm{sec}$ of water presented during the initial portion of the ISI, there was a further $50 \mathrm{sec}$ preceding the presentation of the next flavor in the list.

\section{Results and Discussion}

The mean amount consumed at test, at each serial position, for all three groups is displayed in Figure 2. An inspection of these data reveals that Group $10 \mathrm{sec}$ displayed a strong primacy effect and a somewhat weaker (although numerically present) recency effect; at test, more of the flavors presented at the start of the list were consumed, as compared with those in the center of the list. Group $3.5 \mathrm{sec}$ displayed a serial position function similar to that for Group $10 \mathrm{sec}$ over the first four serial positions. However, there was no sign of a recency effect in this latter group; at test, consumption of the final flavor presented in the initial list was low. In contrast, there was no sign of a primacy effect in Group $60 \mathrm{sec}$, although there was a recency effect in this latter group; at test, consumption of the first flavor presented in the initial list was low.

A two-factor ANOVA (group $\times$ serial position) was conducted on these data and revealed that the main effect of group was not significant $(F<1)$. There was a significant effect of serial position $[F(2,288)=3.58]$, but the interaction between group and serial position narrowly failed to reach conventional levels of significance $[F(8,288)=$ $1.86, .07>p>.06]$. Separate trend tests were conducted on each of the three groups. For Group $10 \mathrm{sec}$, there was only a significant $U$-shaped quadratic trend $[t(24)=2.23]$. This result implies that the first and last flavors presented during the initial list produced greater levels of consumption at test than did the flavors presented in the middle of the initial list. Planned comparisons revealed a significant difference between serial positions one and three $[t(24)=2.19]$, and a difference between serial positions three and five that narrowly failed to reach the conventional level of significance $[t(24)=2.01, .06>p>.05]$. 
For Group $3.5 \mathrm{sec}$, there were no significant trends (all $p s>.10)$. Planned comparisons revealed no significant differences either between serial positions one and three or between serial positions three and five $(p s>.10)$. For Group $60 \mathrm{sec}$, there was a significant linear trend $[t(24)=$ $7.81]$ and a significant quadratic trend $[t(24)=3.56]$. These results imply that the flavors from both ends of the initial list were consumed to a greater extent than the flavors from the middle of the list but that the size of the difference was much greater for the last than for the first stimulus. Planned comparisons revealed no significant difference between serial positions one and three $(p>$ $.10)$, but there was a significant difference between serial positions three and five $[t(24)=5.10]$. Simple effect analyses conducted between the groups revealed a significant difference at serial position one $[F(2,359)=3.15]$ and at serial position five $[F(2,359)=3.06]$.

The results of Experiment 2 suggest that the size of the primacy effect is reduced by increasing the length of the ISI. The two groups with the shorter ISI values (Group $3.5 \mathrm{sec}$ and Group $10 \mathrm{sec}$ ) had similar-sized primacy effects to one another. This finding indicates that the difference in the length of the ISI between these groups was not sufficient to generate significant effects on the primacy effect (this finding replicates that noted in the present Experiment 1). The group with a longer ISI (Group $60 \mathrm{sec}$ ), however, displayed no primacy effect. This result is important, since it confirms the interpretations of the findings reported by Cook et al. (1991) with respect to the influence of ISI length on nonhuman primacy effects; they noted a reduction in primacy when visual stimuli presented to two monkeys had a longer ISI than when they had a shorter ISI.

It should be noted that the retention intervals between the first flavor and test were not equated in the three groups of rats. The longer ISI groups have a longer total retention interval between the first flavor in the list and the test. However, it is unlikely that this aspect of the procedure affected the results, since the total discrepancy was less than 4 min and no effect of retention interval was found on recognition functions across periods of $24 \mathrm{~h}$ by Reed et al. (1996) with the same procedure.

A possible generalization decrement account of these data should also be considered. Generalization decrement may have occurred to a greater extent in the group receiving the short ISI (Group $3.5 \mathrm{sec}$ ) than it did in the groups receiving the longer ISI. The closer presentation of the flavors to one another may have facilitated this process. However, this account assumes that the mouthwash was less effective in Group $3.5 \mathrm{sec}$ than it was in the other two groups. Although this consideration may have exerted some influence over the results in Group $3.5 \mathrm{sec}$, other aspects of the present data imply that generalization decrement will not always lead to a primacy effect; for example, Group $60 \mathrm{sec}$ displayed little primacy effect and yet displayed a recency effect of a size comparable with those for the other groups (if not a little larger). If gen- eralization decrement was always present in this procedure, the primacy effect should always be pronounced, and Group 60 sec should also have displayed such an effect. Thus, in order for a generalization decrement account to be applied to these data, it would have to be applied selectively.

\section{EXPERIMENT 3}

The preceding experiments have shown that primacy and recency effects can be produced with the present procedure and that the primacy effects do not appear to be solely the result of generalization decrement. To a large extent, this validates the present paradigm as an appropriate index of serial position effects, especially because a manipulation known to influence primacy effects with other procedures also exerts similar influences with this procedure. However, before further documentation of these effects is attempted, it seems important to turn to an investigation of one further aspect of the present flavor procedure that may introduce some paradigm-specific influences over the results reported-that is, the nature of the test situation. During the test, the subject has free access to the five flavored liquids that were presented during the initial list. This aspect of the test procedure, potentially, could introduce some confounds into the results obtained. For example, if a subject sampled from only some of the bottles during the test, perhaps because of a position bias, this might affect the results. In addition, if a rat sampled and rejected a number of flavors before coming to one which it recognized, the first flavors sampled during the test would presumably also become familiar over the course of the test session. Given this, the animal may return to those bottles more readily during the test. If this were so, there should be a correlation between the ordinal position of the bottle sampled at test and the amount of that flavor consumed during the test (the earlier the rat is exposed to the flavor, the less neophobia there should be over the course of the test). Although it is difficult to see how these possibilities could systematically influence the results, given the randomization procedures in the test phase, the behavioral dynamics of the task need to be assessed.

\section{Method}

Subjects and Apparatus. Twenty-five male hooded Lister rats (plus 4 animals used in a pilot experiment) were used in this study. The rats were between 9 and 10 months old at the start of the study and had a free-feeding body weight of $500-605 \mathrm{~g}$. The rats were housed and maintained as described in Experiment 1. The apparatus and flavors were the same as those in Experiment 1.

Procedure. The procedure was the same as that described for Group ISI-Water in Experiment 1. A brief pilot experiment $(n=4)$ was conducted to equate the flavors for preference biases. The experimental rats were trained to drink from a syringe. Following this pretraining, each rat received presentation of five flavors. The order of flavor presentation was varied across the 25 rats to control for any remaining flavor preferences. Each flavor occurred at each serial position in the initial list five times. The order of the five flavors 


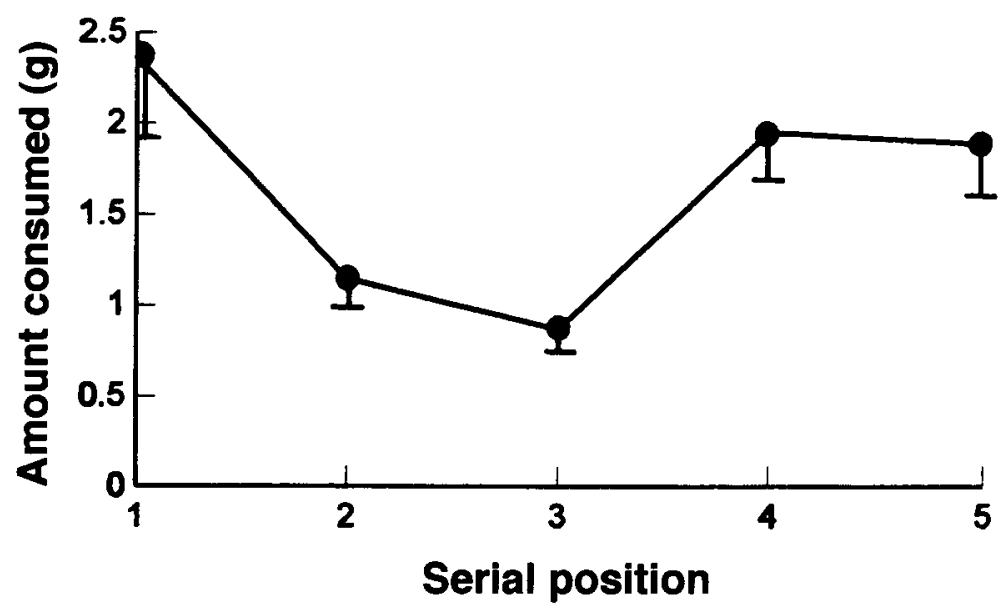

Figure 3. Results from Experiment 3: mean amount of liquid (in grams) consumed during test. Error bars are standard errors of the mean.

was also quasi-random between rats, in that no runs of three flavors were ever given to 2 rats. Prior to the first flavor, the subjects received $0.5 \mathrm{ml}$ of water, delivered over $30 \mathrm{sec}$ by the infusion technique. Between each flavor, the subjects received $0.2 \mathrm{ml}$ of water, delivered over $10 \mathrm{sec}$ by the same infusion technique.

Following exposure, all the rats received a 30 -min retention interval ( $90 \mathrm{sec}$ water plus $28.5 \mathrm{~min}$ without liquid). At test, all five of the bottles were replaced through the front of the subjects' cages. The order in which the bottles were inserted from left to right across the cages was random with respect to the order in which the subjects had experienced the flavors during the exposure phase. The subjects were then allowed $15 \mathrm{~min}$ free access to the solutions. However, in this study the experimenter remained in the test room and recorded the order in which the flavors were sampled by each subject.

\section{Results and Discussion}

Figure 3 shows the mean amount of liquid consumed at test at each serial position in the initial sequence. An inspection of these data reveals that there were pronounced primacy and recency effects. An ANOVA conducted on these data revealed a difference between the positions that was narrowly short of conventional levels of significance $[F(4,96)=2.33, .07>p>.06]$. Trend tests conducted on these data revealed only a significant $U$-shaped quadratic trend $[t(24)=2.36]$. This result implies that the rats consumed, at test, more of the flavors initially presented first and last in the list than they did of the flavors presented in the center of the list. Planned comparisons revealed a significant difference between serial positions one and three $[t(24)=2.67]$ and between serial positions three and five $[t(24)=2.09]$. These data corroborate those presented in the present Experiments 1 and 2 and previously (Reed et al., 1996).

Table 1 shows the number of times at test that the rats sampled each of the items from the initial list as the first bottle sampled at test, as the second bottle sampled at test, and as the third, fourth, and fifth bottles sampled at test. That is, Table 1 shows, for the test session, the number of rats that initially sampled the item that had been pre- sented first in the preceding list, the number that initially sampled the item that had been presented second during the preceding list exposure, and so forth. An inspection of these data reveals that there were no particularly striking deviations from that which could be expected by chance. During the test, the rats were just as likely to sample the first item presented in the preceding list before any of the other items as they were to sample the second, third, fourth, or fifth flavor presented in the preceding list before any of the other flavors at test. Separate chisquare tests were conducted on these data, in order to determine whether the serial position of a flavor in the initial list had an impact on the order of sampling of the flavors during the test. Chi-square tests on the distributions of ordinal sampling during test, for each of the five serial positions during initial list presentations, revealed no significant deviations from chance performance at test (all $p s>.20$ ). There are two reservations that should be noted about the use of this test. First, although testing separately the effect of each of the five serial positions in the initial list on the order of test sampling means that these data subject to each chi-square test are independent (i.e., each rat is only represented once in each data set), it does mean that repeated testing has been performed on these data as a whole. That is, five tests were conducted, which would increase the chances of obtaining a false positive

Table 1

Number of Rats Sampling List Items at Each Ordinal Position During Test

\begin{tabular}{lccccc}
\hline \multirow{2}{*}{$\begin{array}{c}\text { Serial Position } \\
\text { in Initial List }\end{array}$} & \multicolumn{5}{c}{ Ordinal Position of Sampling at Test } \\
\cline { 2 - 6 } & First & Second & Third & Fourth & Fifth \\
\hline One & 4 & 3 & 5 & 8 & 5 \\
Two & 7 & 8 & 3 & 4 & 3 \\
Three & 7 & 8 & 4 & 3 & 3 \\
Four & 3 & 4 & 6 & 4 & 8 \\
Five & 4 & 2 & 7 & 6 & 6 \\
\hline
\end{tabular}


Table 2

Number of Rats Sampling Test Items at Each Spatial Position During Test

\begin{tabular}{lrrrrr}
\hline \multirow{2}{*}{$\begin{array}{c}\text { Spatial Position } \\
\text { in Test Cage }\end{array}$} & \multicolumn{5}{c}{ Ordinal Position of Sampling at Test } \\
\cline { 2 - 6 } & First & Second & Third & Fourth & Fifth \\
\hline One & 11 & 5 & 3 & 1 & 5 \\
Two & 4 & 9 & 6 & 4 & 2 \\
Three & 3 & 4 & 11 & 4 & 3 \\
Four & 2 & 4 & 4 & 10 & 5 \\
Five & 5 & 3 & 1 & 6 & 10 \\
\hline
\end{tabular}

Note-Spatial position one was the leftmost bottle, and spatial position five was the rightmost.

result. Second, the expected number of observations in each of the cells was five, which is only just at a level that would allow for reliable use of chi-square.

There was no significant correlation between the order in which the list items were sampled at test and the subsequent consumption of those items at test $\left[r_{\mathrm{s}}(25)=.016\right.$, $p>$.80]. Thus, ordinal position of the flavor in the initial list had no impact on order of sampling of the flavors at test. Furthermore, order of sampling at test had no relationship to the amount consumed of that flavor at test.

Table 2 shows the order in which the flavors were sampled at test with respect to their spatial position across the cage at test (leftmost bottle $=1$, rightmost bottle $=5$, as the experimenter observed the rat). There were some discernable patterns to these data. For example, more animals than might be expected by chance sampled the flavor on the far left of the array of flavors at test. Chi-square tests were conducted to see whether the spatial position of the bottle in the test cage affected the ordinal sampling of the bottles at test. Separate chi-square tests were conducted on each of the orders of sampling of the bottles as a result of each of the five bottle positions in the cage, to avoid the problems of nonindependence of the data. These tests revealed a significant deviation from chance in the order of sampling for the bottle occupying the extreme left position in the cage $\left[\chi^{2}(4)=11.2, p<.02\right]$. It should be noted, however, that with repeated testing, the appropriate level of significance for these tests, in order to maintain an error rate of $p<.05$, would be $p<.01$.

Inspection of the sequence of sampling revealed that $7 / 25$ rats $(28 \%)$ sampled the flavors in a strict left-to-right sequence. No other sequences of sampling were readily apparent. In order to ascertain whether or not there were differences in the consumption patterns owing to this sampling strategy, the mean level of consumption of the liquids at test was calculated, both for those animals adopting this left-to-right strategy and for those rats not adopting this strategy. The mean consumption of the liquids (with standard errors) by the animals with this leftto-right strategy for serial positions one to five were: $2.07 \mathrm{~g}$ $(0.54), 1.35 \mathrm{~g}(0.32), 0.94 \mathrm{~g}(0.21), 1.14 \mathrm{~g}(0.48)$, and $1.01 \mathrm{~g}$ (0.21), respectively. These scores for the subjects not adopting this strategy were: $2.49 \mathrm{~g}(0.76), 1.07 \mathrm{~g}(0.18), 0.85 \mathrm{~g}$ $(0.16), 2.26 \mathrm{~g}(0.59)$, and $2.23 \mathrm{~g}(0.64)$. Both sets of sub- jects displayed primacy and recency effects, and no discernable effect of strategy was noted on performance. A two-factor ANOVA (strategy $\times$ serial position) was conducted on these data. Neither the main effect of strategy $(p>.10)$ nor the interaction between strategy and serial position $(p>.10)$ proved to be significant in this analysis.

The present data demonstrate that a full serial position function can be generated with this technique and that the behavior of the rat at test, with respect to their sampling of the flavors, does not appear to make a great deal of difference to the type of serial position function obtained. Indeed, apart from a sizable minority of the animals who adopted one particular positional strategy in sampling the flavors during the test session, no particular pattern emerged from these data.

\section{EXPERIMENT 4}

The final experiment was performed to corroborate that increasing the ISI during list presentation serves to reduce the level of the primacy effect. The demonstration that the factors that influence serial recognition in this procedure are similar to those factors that apparently influenced serial recall in other procedures (see Cook et al., 1991) also serves to validate the flavor paradigm. A finding that longer ISIs between items acts to reduce the size of the primacy effect would demonstrate that primacy effects are not an inevitable consequence of the present procedure. To these ends, two groups of rats received five flavors (which were slightly different from those already employed in the experiments reported above). One group of rats had a 10 -sec ISI between items from the list, and the other group had a $60-\mathrm{sec}$ ISI between flavors in the initial list. A strong primacy effect should be noted in the 10 -sec ISI group (replicating the preceding experiments reported here). In contrast, if the previous results reported here were to be replicated, the group with the $60-\mathrm{sec}$ ISI should only demonstrate recency effects.

\section{Method}

Fifty subjects were housed and maintained as described in Experiment 1 . The rats were $10-11$ months old and had a free-feeding body weight of 635-740 g. The apparatus was the same as that in Experiment 1. The procedure was the same as that in Experiment 2 with respect to Group $10 \mathrm{sec}$ and Group $60 \mathrm{sec}$. The five flavors used in this study were lemon, brandy, peppermint, almond, and vanilla. A pilot experiment $(n=10)$, as described in Experiment 1 , was conducted in an attempt to ensure equal consumption of these new flavors.

\section{Results and Discussion}

Figure 4 displays the mean amount consumed at test, for each serial position, for both groups. Inspection of these test data reveals that Group $10 \mathrm{sec}$ consumed greater amounts of the first and last flavors in the initial sequence than of those flavors in the middle of the sequence. That is, both primacy and recency effects were noted for this group. In contrast, during the test phase, Group $60 \mathrm{sec}$ 


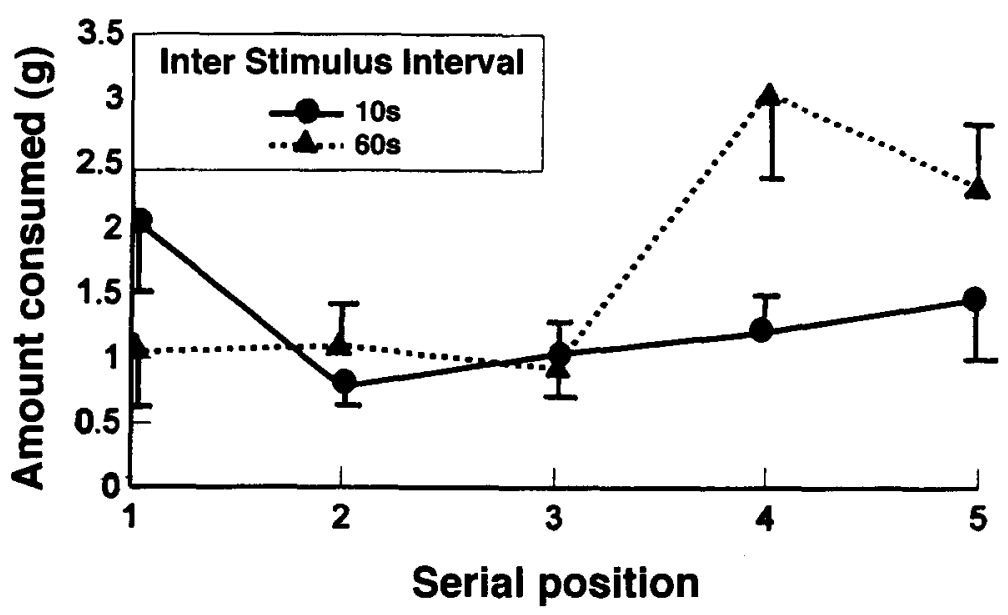

Figure 4. Results from Experiment 4: mean amount of liquid (in grams) consumed during test for both groups. 10s, 10-sec interstimulus interval (ISI); 60s, 60sec ISI. Error bars are standard errors of the mean.

consumed greater amounts of the terminal flavors in the initial list than of the other flavors. That is, only recency effects were noted in these subjects.

A two-factor ANOVA (group $\times$ serial position) revealed a significant main effect of serial position $[F(4,192)=$ $2.95]$ but no main effect of group $(p>.10)$. There was a significant interaction between the two factors $[F(4,192)=$ 2.97]. Separate trend tests were conducted on each of the groups. For Group $10 \mathrm{sec}$, there was only a significant $\mathrm{U}$ shaped quadratic trend $[t(24)=2.09]$. This implies that the rats consumed more of the flavors initially presented at the ends of the initial list than of those presented in the middle of the initial list. Planned comparisons were conducted between both ends of the serial position and the middle position (although it should be noted that the lowest point of the function was serial position two, and these analyses are also included). The analyses for Group $10 \mathrm{sec}$ showed that there were significant differences between serial positions one and three $[t(24)=1.94]$ and between serial positions one and two $[t(24)=2.04]$, but only a marginal difference between serial positions three and five $[t(24)=1.74]$; however, there was a significant difference between serial positions two and five $[t(24)=$ 2.33]. For Group $60 \mathrm{sec}$, only an increasing linear trend was significant $[t(24)=3.04]$. Planned comparisons revealed no significant differences between serial positions one and three $(t<1$; similarly, $t<1$ for serial positions one and two), but there was a significant difference between serial positions three and five $[t(24)=2.45]$ and, similarly, between serial positions two and five $[t(24)=$ $2.12]$. An analysis of the simple main effect of group revealed that Group $10 \mathrm{sec}$ consumed more at serial position one than did Group $60 \mathrm{sec}[F(1,239)=2.81]$ but that there was no difference at serial position five $(p s>.10)$.

These data corroborated those obtained from the present Experiment 2 but did so by using a different set of flavors than did the former experiment. Increasing the
ISI between items in the initial list decreased the size of the primacy effect that was obtained. This result suggests that the present procedure is influenced by factors similar to those noted in other procedures (cf. Cook et al., 1991) and that a primacy effect is not a necessary result of generalization decrement with this procedure.

\section{GENERAL DISCUSSION}

In the present experiments, rats' memory for serially presented, nonspatial items was investigated. In all the experiments, primacy and recency effects were generated. That robust primacy effects can be generated in nonhumans is not a novel finding (Buffalo, D. Gaffan, \& Murray, 1994; Roberts \& Kraemer, 1981; Wright et al., 1985). Neither is the generation of primacy effects in rats novel (see Bolhuis \& Van Kampen, 1988; DiMattia \& Kesner, 1984; Harper et al., 1993). However, that a primacy effect in rats can be demonstrated in a nonspatial task is of some interest, given recent discussions of this topic (see Deacon \& Rawlins, 1995; E. A. Gaffan, 1992; Reed, 1994). Of course, the present results do not speak to the question of whether such effects are demonstrable in other nonspatial procedures, such as those concerned with visual modalities (cf. Deacon \& Rawlins, 1995; Reed et al., 1991). It may be that the gustatory/olfactory cues utilized here are served by specialized mechanisms that do not operate for stimuli from other modalities. However, the fact that a similar manipulation of the ISI during initial list presentation resulted in effects in this study, which used flavors with rats (Experiments 2 and 4), that were similar to those for monkeys exposed to visual stimuli in the report by Cook et al. (1991) suggests that the above view is unparsimonious, at best.

Experiment 1 tested whether generalization decrement could have contributed to primacy effects. Such an explanation suggests that successive exposure to flavors would 
produce increasing amounts of contamination of the subsequently presented flavors by those flavors that preceded them in the initial list. Thus, for example, the last flavor experienced in the list would be a compound of all the previously presented flavors. During the test, this phenomenon would make recognition of all flavors except the first flavor presented in the list difficult, since, in effect, none of the other flavors would have been experienced previously. Given this, the neophobic response to all but the first flavor would be high during the test.

That such an effect may contribute to the results generated by the present procedure is suggested by two pieces of evidence. First, when water was not presented between the flavors during initial exposure, test consumption of the items presented later in the list was reduced, a finding consistent with the above view (see Experiment 1). Second, an inspection of the data from the previous report using the flavor paradigm (Reed et al., 1996) shows that the relationship of primacy and recency to retention interval is not that normally seen in studies of memory. Neither effect is observed with the present procedure at nominal zero-retention intervals, but both effects develop with longer intervals. There is some evidence to suggest that generalization between items is greatest immediately after their presentation and that generalization declines with the passage of time after their presentation (see Hall, 1991). The generalization decrement phenomenon would predict the above pattern of serial position effects with respect to the effect of retention interval.

On the other hand, there are also three strong pieces of evidence suggesting that generalization decrement is not the sole mechanism responsible for the primacy effect. First, presentation of water between the list items produced recency effects (Experiment 1). This suggests that the presentation of water between the flavors had an impact on serial contamination. Second, the recency effect implies that serial position effects cannot solely be attributed to generalization decrement, because this view does not predict that a recency effect would ever occur. Third, the results from the present Experiments 2 and 4 suggest that generalization decrement cannot be the sole explanation of the primacy effect, because little (if any) primacy was noted in the rats' performance at test when a longer ISI was used between the initially presented list items. Thus, primacy is not the automatic outcome of the present procedure.

The results from Experiments 2 and 4 also suggest that nonhuman memory for serially presented items is subject to different influences than is human memory for serially presented verbal material. In these experiments, as in the report by Cook et al. (1991), increasing the ISI between items in the list attenuated the primacy effect. However, in studies of human memory, manipulation of the ISI leads to enhanced memory for the initially presented items (Glazner \& Cunitz, 1966). In humans, this result is taken to reflect the operation of a rehearsal mechanism, by which the first items presented in the list gain access to a longterm memory store. The longer the interval between suc- cessive presentation of the list items, the more rehearsal can occur prior to the interruption of the process by the presentation of the next item in the list (see also Reed, 1998; Wright et al., 1990). That the manipulation of the ISI between list items does not result in the same enhancement of the primacy effect in nonhumans has been taken to reflect the absence of a rehearsal mechanism in nonhuman memory for serially presented items. The results obtained from the present Experiments 2 and 4 do nothing to disconfirm this suggestion.

An alternative to the conceptualization of these results in terms of memory is that they may reflect the operation at the time of test of some hedonic process; that is, all the flavors could have been equally well remembered, but some could have been preferred to others. The primacy effect may be due to positive hedonic properties acquired by the first flavor presented in the sequence. These properties may arise because the initial flavor in the sequence is also among the first liquids that occur after a period of deprivation. Positive hedonic properties associated with a flavor may cause the animal to approach and consume this flavor at test. The last flavor may acquire such properties through its contiguity with satiation, which could occur after presentation of all the liquid given in the study. The notion that the hedonic (or associative) value of a stimulus exerts an influence over serial position effects has been explored in other contexts (see Wixted, 1986; Wynne, 1995). It may be that some modified version of this view could also be extrapolated to the present context.

The present results should not necessarily be taken to support the notion that there is a species difference in the mechanisms that govern memory for serially presented items across all memory paradigms. Just as likely is the notion that the present results reflect a difference between the processing given to different types of stimulus materials. It could be, for example, that human memory for nonverbal stimuli is affected in ways similar to those for nonhuman memory for such material. It is clear that humans do show both primacy and recency effects for nonverbal material presented in a list (Neath, 1993; Reed, 2000; Roberts \& Kraemer, 1981). What is less clear is whether human memory for serially presented nonverbal items is subject to the same influences as human memory for serially presented verbal items. The results of two separate experiments suggest that the ability to name a stimulus presented in a list plays a critical role in determining the mechanisms that will govern the generation of serial position effects (see Reed, 2000; Wright et al., 1990). The role of language in mediating memory for serially presented items is yet to be well explored, and it would be unwise to speculate further, on the basis of the present data.

\section{REFERENCES}

Atkinson, R. C., \& Shiffrin, R. M. (1968). Human memory: A proposed system and its control processes. In K. W. Spence \& J. T. Spence (Eds.), The psychology of learning and motivation: Advances in research and theory (Vol. 2, pp. 89-195). New York: Academic Press. 
Baddeley, A., Papagno, C., \& Andrade, J. (1993). The sandwich effect: The role of attentional factors in serial recall. Journal of Experimental Psychology: Learning, Memory, \& Cognition, 19, 862-870.

Bolmuis, J. J., \& VAN Kampen, H. S. (1988). Serial position curves in spatial memory of rats: Primacy and recency effects. Quarterly Journal of Experimental Psychology, 40B, 135-149.

Buffalo, B., Gaffan, D., \& Murray, E. A. (1994). A primacy effect in monkeys when list position is relevant. Quarterly Journal of Experimental Psychology, 47B, 353-369.

COOK, R. G., WRIGHT, A. A., \& SANDS, S. F. (1991). Interstimulus interval and viewing time effects in monkey list memory. Animal Learning \& Behavior, 19, 153-163.

DeACoN, R. M. J., \& Rawlins, J. N. P. (1995). Serial position effects and duration of memory for nonspatial stimuli in rats. Journal of Experimental Psychology: Animal Behavior Processes, 21, 285-292.

DiMattia, B. V., \& KeSNER, R. P. (1984). Serial position curves in rats: Automatic versus effortful information processing. Journal of Ex perimental Psychology: Animal Behavior Processes, 10, 557-563.

GAFFAN, D. (1983). A comment on primacy effects in monkeys' memory for lists. Animal Learning \& Behavior, 11, 144-145.

GafFan, E. A. (1992). Primacy, recency, and the variability of data in studies of animals' working memory. Animal Learning \& Behavior, 20, 240-252.

Glazner, M., \& Cunitz, A. R. (1966). Two storage mechanisms in free-recall. Journal of Verbal Behavior \& Verbal Learning, 5, 351-360.

HaLl, G. (1991). Perceptual and associative learning. Oxford: Oxford University Press, Clarendon Press.

Harper, D. N., Mclean, A. P., \& Dalrymple-Alford, J. C. (1993). List item memory in rats: Effect of delay and delay task. Journal of Experimental Psychology: Animal Behavior Processes, 19, 307-316.

Johnson, G. J. (1991). A distinctiveness model of serial learning. Psychological Review, 98, 204-217.

MaCPhaIL, E. M. (1980). Short-term visual recognition in pigeons. Quarterly Journal of Experimental Psychology, 32B, 521-538.

NeATH, I. (1993). Contextual and distinctiveness processes and the serial position function. Journal of Memory \& Language, 32, 820-840.

REED, P. (1994). Less than expected variance in studies of serial position effects is not a sufficient reason for caution. Animal Learning \& Behavior, 22, 224-230.

REED, P. (1998). Absence of backward scan mechanism triggered by salient stimuli in human serial list learning. Learning \& Motivation, 29, 133-151.

REED, P. (2000). Serial position effects in human olfactory memory. Journal of Experimental Psychology: Learning, Memory, \& Cognition, 26, 411-422.

Reed, P., Chih-TA, T., Aggleton, J. P., \& Rawlins, J. N. P. (1991). Primacy, recency, and the von Restorff effect in rats' nonspatial recognition memory. Journal of Experimental Psychology: Animal Behavior Processes, 17, 36-44.

Reed, P., Croft, H., \& Yeomans, M. (1996). Rats' memory for serially presented novel flavours: Evidence for non-spatial primacy effects. Quarterly Journal of Experimental Psychology, 49B, 174-187.

REED, P., \& Richards, A. (1996). The von Restorff effect in rats (Rattus norvegicus). Journal of Comparative Psychology, 110, 193-198.

RoberTs, W. A., \& KraEmer, P. J. (1981). Recognition memory for lists of visual stimuli in monkeys and humans. Animal Learning \& Behavior, 9, 587-594.

SANDS, S. R., \& WRIGHT, A. A. (1980). Primate memory: Recognition of serial list items by a rhesus monkey. Science, 209, 938-939.

SCHMIDT, S. R. (1991). Can we have a distinctive theory of memory? Memory \& Cognition, 19, 523-542.

Thompson, R. K. R., \& Herman, L. M. (1977). Memory for lists of sounds by the bottlenosed dolphin: Convergence of memory processes with humans? Science, 195, 501-503.

WIXTED, J. T. (1986). Nonhuman short-term memory: A quantitative reanalysis of selected findings. Journal of the Experimental Analysis of Behavior, 52, 409-426.

Wright, A. A., Cook, R. G., Rivera, J. J., \& Shyan, M. R. (1990). Naming, rehearsal, and interstimulus interval effects in memory processing. Journal of Experimental Psychology: Learning, Memory, \& Cognition, 16, 1043-1059.

Wright, A. A., Santiago, H. C., Sands, S. F., Kendrick, F. F., \& Cook, R. G. (1985). Memory processing of serial lists by pigeons, monkeys, and people. Science, 229, 287-289.

WyNnE, C. D. L. (1995). Reinforcement accounts for transitive inference performance. Animal Learning \& Behavior, 23, 207-217.

(Manuscript received May 11, 1999;

revision accepted for publication January 12,2000 .) 